\title{
Superluminal and ultraslow light propagation in optomechanical systems
}

\author{
Devrim Tarhan, ${ }^{1, *}$ Sumei Huang, ${ }^{2}$ and Özgür E. Müstecaplığ ${ }^{3}{ }^{3}$ \\ ${ }^{1}$ Department of Physics, Harran University, Osmanbey Yerleşkesi, Şanlı urfa, 63300, Turkey \\ ${ }^{2}$ Department of Physics, University of California, Merced, California 95343, USA \\ ${ }^{3}$ Department of Physics, Koç University, Sartyer, Istanbul, 34450, Turkey
}

(Received 26 October 2012; published 22 January 2013)

\begin{abstract}
We consider an optomechanical double-ended cavity under the action of a coupling laser and a probe laser in an electromagnetically induced transparency configuration. It is shown how the group delay and advance of the probe field can be controlled by the power of the coupling field. In contrast to single-ended cavities, which only allow for superluminal propagation, the possibility of both superluminal and subluminal propagation regimes is found. The magnitudes of the group delay and the advance are calculated to be $\sim 1 \mathrm{~ms}$ and about $-2 \mathrm{~s}$, respectively, at a very low pumping power of a few microwatts. In addition, the interaction of the optomechanical cavity with a time-dependent probe field is investigated for controlled excitations of mirror vibrations.
\end{abstract}

DOI: 10.1103/PhysRevA.87.013824

PACS number(s): 42.50.Gy, 42.50.Ct, 42.50.Wk

\section{INTRODUCTION}

The demonstration of an ultraslow group velocity $v_{g}$ of light [1] in ultracold atoms by electromagnetically induced transparency (EIT) [2] has inspired appealing applications [3-8]. Besides the slow light, superluminal phenomena $\left(v_{g}>\right.$ $c$ or $v_{g}$ is negative) were observed in atomic cesium gas $[9,10]$ and in alexandrite crystal [11]. Slow and superluminal light has also been observed in optomechanical systems [12] whose superior delay and advancement times, smaller dimensions, and less demanding thermal requirements make them attractive for quantum-optomechanical memory and classical signal processing applications [13-22]. Recent proposals, such as an optomechanical cavity with a Bose-Einstein condensate (BEC) [23] and a one-sided cavity with a nanomechanical mirror (NMM) [24], which was recently demonstrated [21], are promising but either too costly and difficult to implement [23] or not sufficiently flexible enough to realize both superluminal and slow light effects simultaneously $[21,24]$. The analog of electromagnetically induced transparency has been demonstrated very recently in a room-temperature cavity optomechanics setup formed by a thin, semitransparent membrane within a Fabry-Perot cavity [25]. We address the question of how more controllable and simpler optomechanical systems that can simultaneously exhibit larger delay and advancement times can be realized.

In this work, we investigate the time delay of the weak probe field at the probe resonance in a double-ended high-quality cavity with a moving NMM under the action of a coupling laser. We find that the group delay can be controlled by the power of the coupling field. The time delay is positive, which corresponds to ultraslow light propagation (subluminal propagation), when there is a strong coupling between the nano-oscillator and the cavity. In contrast to single-ended cavities, which only allow for superluminal propagation [26], the possibility of both superluminal and subluminal propagation regimes is found. The magnitude of the group delay is $\sim 2 \mathrm{~ms}$ at a very low pumping power of a few microwatts. The

\footnotetext{
*dtarhan@harran.edu.tr
}

transmission group delay that we have found is larger than the group delay in a coupled BEC-cavity system [23], which is a costly and difficult system to implement. In addition, we show that it is possible to control the vibrational excitations of the NMM with a time-dependent probe field.

The organization of this paper is as follows. In Sec. II we describe our physical optomechanical system and EIT configuration. Quantities such as group delay and advancement times are redefined there as well. The results are given in Sec. III in two parts. The first part is dedicated to the case of constant pump and probe fields, while the other one focuses on the case of time-dependent fields. Our conclusion is given in Sec. IV.

\section{MODEL SYSTEM}

We consider the classical probe field $\varepsilon_{p}$ and calculate the response of the cavity optomechanical system to the probe field in the presence of the coupling field $\varepsilon_{c}$. The nanomechanical oscillator of frequency $\omega_{m}$ is coupled to a Fabry-Pérot cavity via radiation pressure effects [14]. In a Fabry-Pérot cavity, both mirrors have equal reflectivity. We use a configuration in which a partially transparent NMM is in the middle of a cavity that is bounded by two high-quality mirrors, as shown in Fig. 1. The system is driven by a coupling field of frequency $\omega_{c}$, and the probe field has frequency $\omega_{p}$.

The Hamiltonian of this system is given by

$$
\begin{aligned}
H= & \hbar\left(\omega_{0}-\omega_{c}\right) c^{\dagger} c+\hbar g c^{\dagger} c q+\frac{p^{2}}{2 m}+\frac{1}{2} m \omega_{m}^{2} q^{2} \\
& +i \hbar \varepsilon_{c}\left(c^{\dagger}-c\right)+i \hbar\left(c^{\dagger} \varepsilon_{p} e^{-i \delta t}-c \varepsilon_{p}^{*} e^{i \delta t}\right),
\end{aligned}
$$

where $\delta=\omega_{p}-\omega_{c}, g=-\omega_{c} / L$ is the coupling constant between the cavity field and the movable mirror [27], and $c$ and $c^{\dagger}$ are the annihilation and creation operators of the photons of the cavity field, respectively. The momentum and position operators of the nanomechanical oscillator are $p$ and $q$, respectively. The amplitude of the pump field is $\varepsilon_{c}=\sqrt{2 \kappa P_{c} / \hbar \omega_{c}}$, with $P_{c}$ being the pump power.

Heisenberg equation of motion for the coupled cavitymirror system is written and the damping rate $2 \kappa$ is added phenomenologically to represent the loss at the cavity mirrors. 


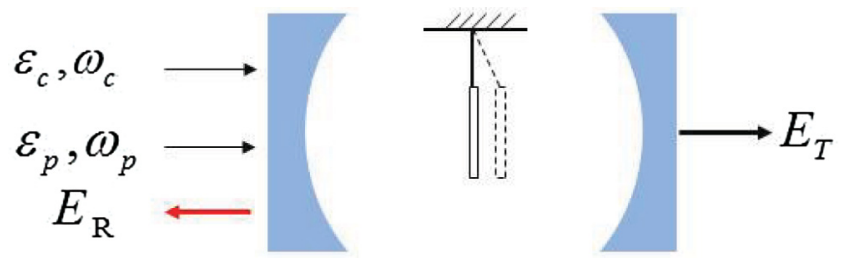

FIG. 1. (Color online) Schematic of a double-ended cavity with a moving nanomechanical mirror, adapted from Ref. [14].

The system is examined in the mean-field limit [24]:

$$
\begin{aligned}
\langle\dot{q}\rangle & =\frac{\langle p\rangle}{m}, \\
\langle\dot{p}\rangle & =-m \omega_{m}^{2}\langle q\rangle-\hbar g\left\langle c^{\dagger}\right\rangle\langle c\rangle-\gamma_{m}\langle p\rangle, \\
\langle\dot{c}\rangle & =-\left[2 \kappa+i\left(\omega_{0}-\omega_{c}+g\langle q\rangle\right)\right]\langle c\rangle+\varepsilon_{c}+\varepsilon_{p} e^{-i \delta t}, \\
\left\langle\dot{c}^{\dagger}\right\rangle & =-\left[2 \kappa-i\left(\omega_{0}-\omega_{c}+g\langle q\rangle\right)\right]\left\langle c^{\dagger}\right\rangle+\varepsilon_{c}+\varepsilon_{p}^{*} e^{i \delta t} .
\end{aligned}
$$

The linear-response solution is developed analytically using the ansatz [28],

$$
\begin{aligned}
& q(t)=q_{0}+q_{+} \varepsilon_{p} e^{-i \delta t}+q_{-} \varepsilon_{p}^{*} e^{i \delta t}, \\
& p(t)=p_{0}+p_{+} \varepsilon_{p} e^{-i \delta t}+p_{-} \varepsilon_{p}^{*} e^{i \delta t}, \\
& c(t)=c_{0}+c_{+} \varepsilon_{p} e^{-i \delta t}+c_{-} \varepsilon_{p}^{*} e^{i \delta t},
\end{aligned}
$$

where $q_{0}, p_{0}$, and $c_{0}$ are the zeroth-order solutions, while the next terms corresponds to the first-order solutions in the probe field amplitude. By inserting Eq. (3) into the Heisenberg equation of motion we first obtain the steady-state solutions $c_{0}=\varepsilon_{c} /(2 \kappa+i \Delta)$ and $q_{0}=-\hbar g\left|c_{0}\right|^{2} / m \omega_{m}^{2}$. Using them, the first-order solutions are analytically determined to be

$$
c_{+}(\delta)=\frac{m\left(\delta^{2}-\omega_{m}^{2}+i \gamma_{m} \delta\right)[2 \kappa-i(\Delta+\delta)]-i \alpha}{m\left(\delta^{2}-\omega_{m}^{2}+i \gamma_{m} \delta\right)\left[(2 \kappa-i \delta)^{2}+\Delta^{2}\right]+2 \Delta \alpha},
$$

where $\Delta=\omega_{0}-\omega_{c}+g q_{0}$ is the effective detuning and $\alpha=$ $\hbar g^{2}\left|c_{0}\right|^{2}$. Here $\left|c_{0}\right|^{2}$ is the resonator intensity, and $q_{0}$ is the steady-state position of the movable mirror.

We can write the output field as $\varepsilon_{\text {out }}(t)=\varepsilon_{\text {out } 0}+$ $\varepsilon_{\text {out }+} \varepsilon_{p} e^{-i \delta t}+\varepsilon_{\text {out }-} \varepsilon_{p}^{*} e^{i \delta t}$ [28]. Inserting this to the inputoutput relation and comparing the coefficient of $\varepsilon_{p} e^{-i \delta t}$, we get the probe response $\left(\varepsilon_{\text {out }+}+1\right)=2 \kappa c_{+}$. The reflection and the transmission of the probe response are denoted by $\varepsilon_{R}$ and $\varepsilon_{T}$, respectively. The reflection and transmission of the output field are determined by $E_{R}=\varepsilon_{R} \varepsilon_{p} e^{-i \omega_{p} t}$ and $E_{T}=\varepsilon_{T} \varepsilon_{p} e^{-i \omega_{p} t}$, respectively. $\varepsilon_{T}=2 \kappa c_{+}(\delta)$ is the transmitted component and $\varepsilon_{R}=2 \kappa c_{+}(\delta)-1$ is the reflected component of the probe field. The amplitude of the transmission output field is $E_{T}=$ $|T| \varepsilon_{p} \exp \left[i \phi\left(\omega_{p}\right)\right]$.

If we expand $\phi\left(\omega_{p}\right)$ around $\bar{\omega}$ to first order,

$$
\phi\left(\omega_{p}\right)=\phi(\bar{\omega})+\left.\left(\omega_{p}-\bar{\omega}\right) \frac{\partial \phi}{\partial \omega_{p}}\right|_{\bar{\omega}}
$$

the transmitted probe pulse can be expressed as $|T| \varepsilon_{p} e^{-i \omega_{p} t} e^{i \phi(\bar{\omega})} e^{i\left(\omega_{p}-\bar{\omega}\right) \frac{\partial \phi}{\partial \omega_{p}} \mid \bar{\omega}}$, where $\phi(\bar{\omega})=0$ at resonance. Combining with $e^{-i \omega_{p}(t-\tau)}$, the transmitted probe pulse peaks at $t=\tau$, where $\tau$ is the pulse delay, which can defined as

$$
\tau=\left.\left[\frac{\partial \phi}{\partial \omega_{p}}\right]\right|_{\bar{\omega}} .
$$

The phase of the output field can be found as

$$
\phi=\frac{1}{2 i} \ln \left(\frac{\varepsilon_{T}}{\varepsilon_{T}^{*}}\right) .
$$

The time delays of the transmission and reflection pulses can be determined by

$$
\tau_{T}=\left.\operatorname{Im}\left[\frac{1}{\varepsilon_{T}} \frac{\partial \varepsilon_{T}}{\partial \omega_{p}}\right]\right|_{\bar{\omega}}, \quad \tau_{R}=\left.\operatorname{Im}\left[\frac{1}{\varepsilon_{R}} \frac{\partial \varepsilon_{R}}{\partial \omega_{p}}\right]\right|_{\bar{\omega}} .
$$

\section{RESULTS AND DISCUSSION}

In our calculations, we use the following parameters [19]: length of the cavity $L=6.7 \mathrm{~cm}$, wavelength of the laser $\lambda=2 \pi c / \omega_{c}=1064 \mathrm{~nm}, m=40 \mathrm{ng}, \omega_{m}=2 \pi \times 134 \mathrm{kHz}$, $\gamma=0.76 \mathrm{~Hz}, \kappa=\omega_{m} / 10$, mechanical quality factor $Q=$ $1.1 \times 10^{6}$, and $\Delta=\omega_{m}$. The real and the imaginary parts of $\varepsilon_{T}=2 \kappa c_{+}$represent the absorptive and dispersive behaviors, respectively.

\section{A. Constant pump and probe fields}

We show the real and the imaginary parts of $\varepsilon_{T}$ in Fig. 2. Under the conditions of electromagnetically induced transparency in the mechanical system contained in a highquality cavity the system gives rise to dispersion, which leads to ultraslow propagation of the probe field [14].

The phase is determined by Eq. (7) and $\varepsilon_{T}$, and the result is plotted in Fig. 3 as a function of the scaled dimensionless frequency $\delta / \omega_{m}$ for input coupling laser power $P_{c}=1 \mu \mathrm{W}$.

In the case of no coupling field, $g=0$, the delay time becomes $\tau_{0}=1.48 \mu \mathrm{s}$. The coupling reverses the behavior of the system, and the group delay becomes positive. We plot the group delay $\tau$ as a function of the pump power in Figs. 4 and 5, which show the group delay $\tau$ as a function of the pump power $P_{c}$. The group delay decreases with increasing power of the coupling field. The probe pulse delay can be tuned by calibrating the pump power in the probe resonance $\left(\delta=\omega_{m}\right)$. The pump power that we have used in

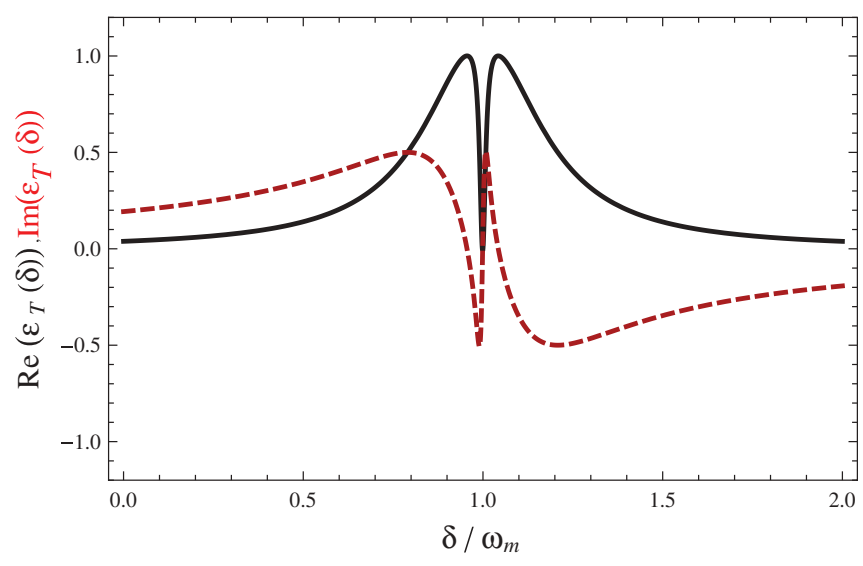

FIG. 2. (Color online) The real (black solid line) and the imaginary (red dashed line) parts of $\varepsilon_{T}(\delta)$ as a function of $\delta$ for an input coupling laser power of $P_{c}=5 \mu \mathrm{W}$. The parameters used are length of the cavity $L=6.7 \mathrm{~cm}$, wavelength of the laser $\lambda=$ $2 \pi c / \omega_{c}=1064 \mathrm{~nm}, m=40 \mathrm{ng}, \omega_{m}=2 \pi \times 134 \mathrm{kHz}, \gamma=0.76 \mathrm{~Hz}$, $\kappa=\omega_{m} / 10$, mechanical quality factor $Q=1.1 \times 10^{6}$, and $\Delta=\omega_{m}$. 


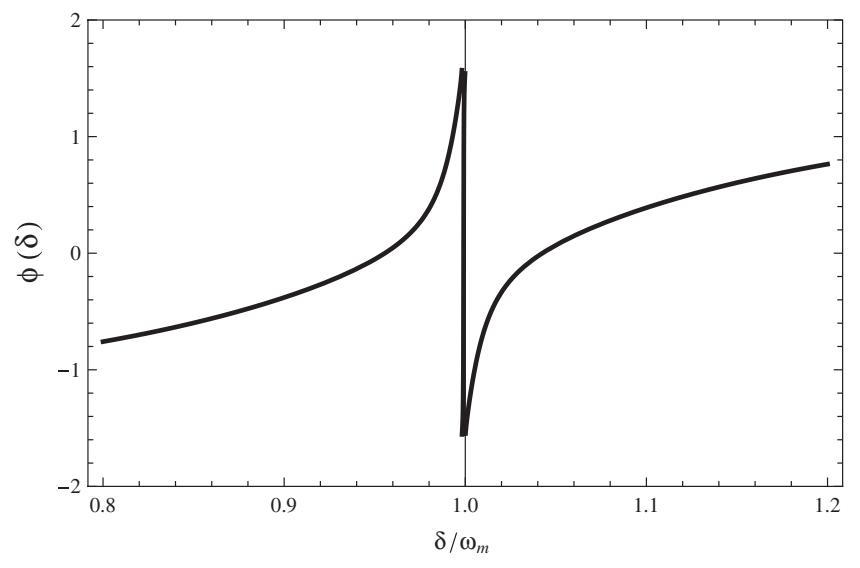

FIG. 3. Phase as a function of frequency $\delta$ for input coupling laser power $P_{c}=1 \mu \mathrm{W}$. The parameters are the same as in Fig. 2 .

Figs. 4 and 5 is on the order of $0.1-5 \mu \mathrm{W}$. In Fig. 4, the group delays are negative, which means that the reflected probe field is a superluminal light. In Fig. 5 the group delays are positive; as a result the slow light effect can be observed. This corresponds to a subluminal situation. We find large positive group delays of order $2 \mathrm{~ms}$ in a Fabry-Pérot cavity under the action of a coupling laser and a probe laser. The physics of subluminal or superluminal light propagation in a double-ended cavity optomechanical system is associated with the interaction of the NNM and the cavity field.

We plot the reflection $R(\delta)=\left|2 \kappa c_{+}-1\right|^{2}$ and transmission spectrums $T(\delta)=\left|2 \kappa c_{+}\right|^{2}$ of the probe field in Figs. 6 and 7 , respectively. The width of the transparency window of EIT is given by [14]

$$
\Gamma\left(P_{c}\right)=\frac{\gamma_{m}}{2}+\frac{\alpha\left(P_{c}\right)}{4 m \omega_{m} \kappa},
$$

where $\alpha\left(P_{c}\right)=\hbar g^{2}\left|c_{0}\right|^{2}$. The EIT width changes with the power in a linear manner, as shown in Fig. 8.

\section{B. Time-dependent probe field}

We now consider the time-dependent, pulsed probe field. As the dynamics of the optomechanical system is associated with the normal modes of the cavity field and the mirror vibrations,

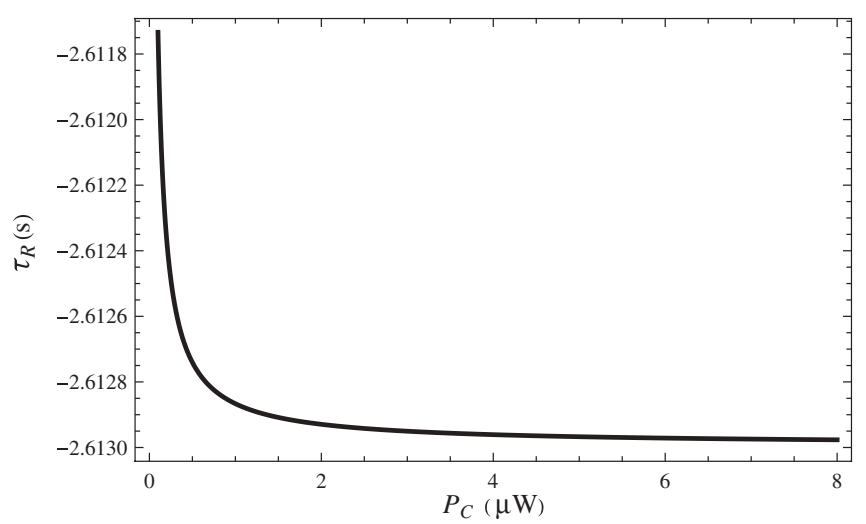

FIG. 4. Advance of the reflected probe field as a function of the pump power in the presence of the coupling field. All parameters are the same as those in Fig. 2.

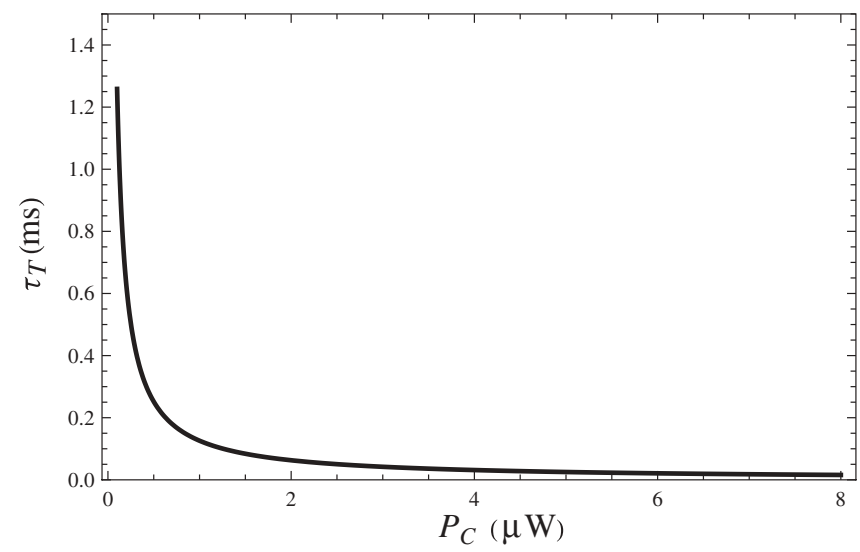

FIG. 5. Group delay of the transmitted probe field as a function of the pump power in the presence of the coupling field. All parameters are the same as those in Fig. 2.

it is natural to expect that the oscillation modes of the mirror can be controlled with temporal profile of the optical fields. We examine particularly pulses with duration much less than the characteristic time of mirror oscillations. The effect of such pulses can be interpreted as if the mirror oscillator is kicked by the optical pulses in sudden perturbations. We find situations with both robust excitations of mirror motional modes, where the mirror is simply displaced without oscillations, and periodic excitations, where the mirror vibrates.

Now we use the following ansatz in terms of time dependence in order to obtain resonator-mirror coupled equations:

$$
\begin{aligned}
& q(t)=q_{0}(t)+q_{+}(t) e^{-i \delta t}+q_{-}(t) e^{i \delta t}, \\
& p(t)=p_{0}(t)+p_{+}(t) e^{-i \delta t}+p_{-}(t) e^{i \delta t}, \\
& c(t)=c_{0}(t)+c_{+}(t) e^{-i \delta t}+c_{-}(t) e^{i \delta t} .
\end{aligned}
$$

Since the probe field is very weak, the force acting on the mirror exerted by the time-dependent probe field is negligible, so we assume $\dot{p}_{+}(t)=0$. Moreover, we assume that the system is working in the resolved sideband limit $\left(\kappa<\omega_{m}\right)$ and the Stokes field generated by the interaction of the

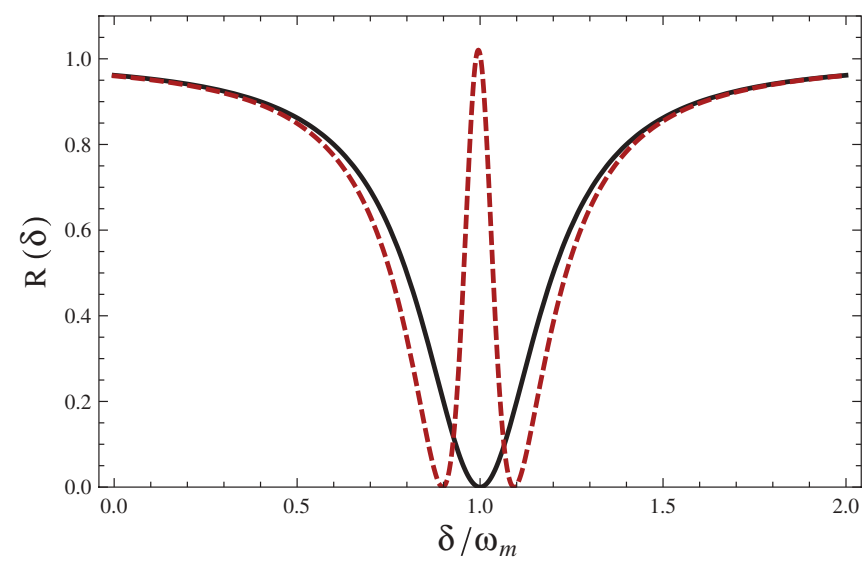

FIG. 6. (Color online) The reflection spectrum $R(\delta)$ as a function of normalized frequency. $P_{c}=0$ solid, $5 \mu \mathrm{W}$ (dashed). All parameters are the same with those of Fig. 2. 


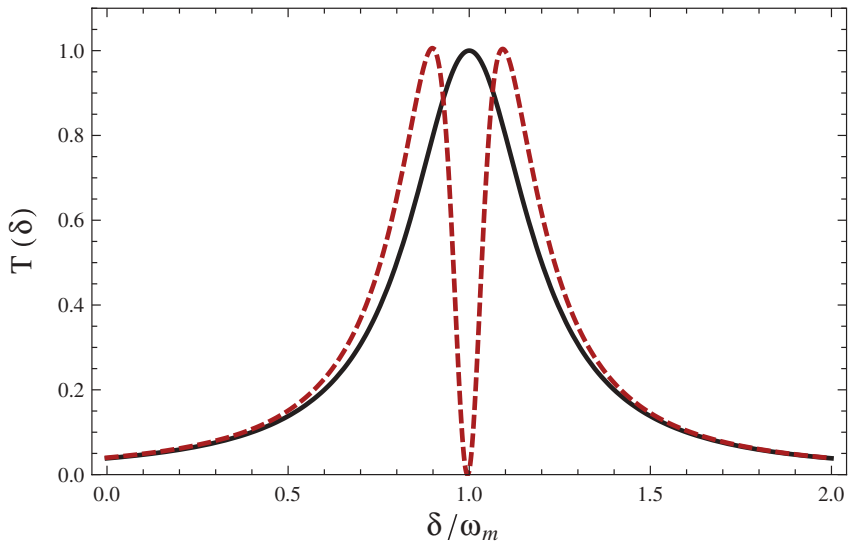

FIG. 7. (Color online) The transmission spectrum $T(\delta)$ as a function of normalized frequency. $P_{c}=0$ solid, $5 \mu \mathrm{W}$ (dashed). All parameters are the same with those of Fig. 2.

coupling field with the mirror is very small; therefore we assume $\dot{c}_{-}^{*}(t)=0$. Substituting Eq. (10) into Eq. (2), we obtain

$$
\begin{aligned}
& \frac{d q_{+}}{d t}=\frac{1}{s}\left\{d-i \hbar g^{2}\left|c_{0}\right|^{2}\right\} q_{+}-\left[\frac{\hbar g}{m\left(\gamma_{m}-i \delta\right)} c_{0}^{*}\right] c_{+}, \\
& \frac{d c_{+}}{d t}=-[2 \kappa+i(\Delta-\delta)] c_{+}-i g c_{0} q_{+}+\varepsilon_{p}(t),
\end{aligned}
$$

where $s=m\left(\gamma_{m}-i \delta\right)[2 \kappa-i(\Delta+\delta)], d=m[2 \kappa-i(\Delta+$ $\delta)]\left(i \delta \gamma_{m}-\delta^{2}-\omega_{m}^{2}\right)$, and $\Delta=\omega_{0}-\omega_{c}+g q_{0}$ is the effective detuning. The zeroth-order solutions are $c_{0}=\varepsilon_{c} /(2 \kappa+i \Delta)$ and $q_{0}=-\hbar g\left|c_{0}\right|^{2} / m \omega_{m}^{2}$. Equation (11) describes the coupled, normal-mode excitations of mirror and optical mode propagation of the probe field in a nanomechanical system. One can solve Eq. (11) by introducing the matrix notation

$$
\begin{gathered}
\vec{V}=\left(\begin{array}{l}
q_{+} \\
c_{+}
\end{array}\right), \\
\widetilde{M}=\left(\begin{array}{cc}
A & B \\
C & D
\end{array}\right), \\
\vec{F}=\left(\begin{array}{c}
0 \\
\varepsilon_{p}(t)
\end{array}\right),
\end{gathered}
$$

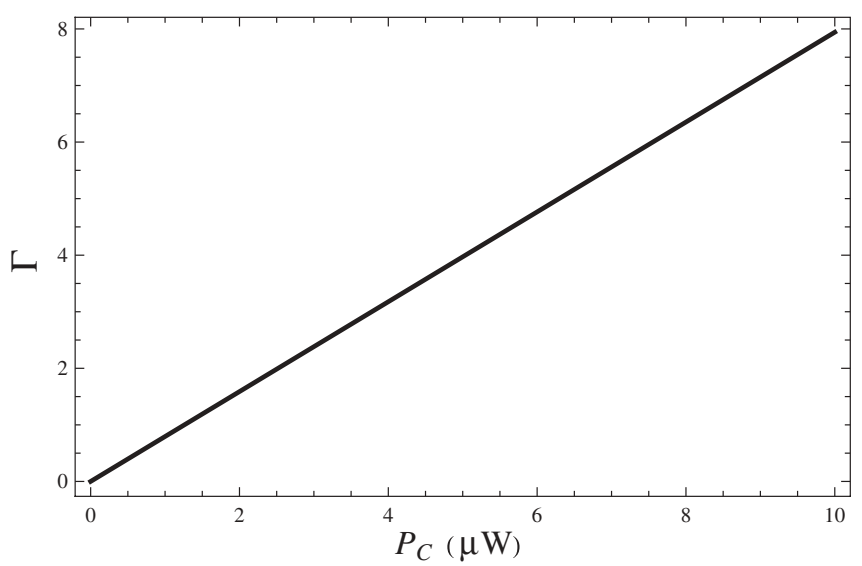

FIG. 8. EIT width $\Gamma\left(P_{c}\right)$ as afunction of power $P_{c} \Gamma$ is normalized by $1 \times 10^{4} 1 / \mathrm{s}$. All parameters are the same with those of Fig. 2 .

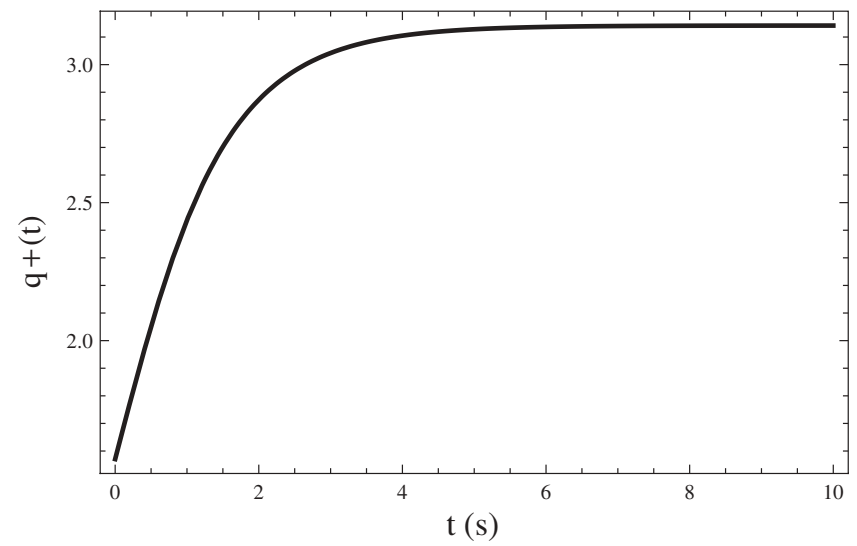

FIG. 9. $q_{+}(t)$ as a function of time with $\Delta=\omega_{m}$. All parameters are the same as those in Fig. 2.

where $A=\left(-d+i \hbar g^{2}\left|c_{0}\right|^{2}\right) / s, B=\hbar g c_{0}^{*} / m\left(\gamma_{m}-i \delta\right), C=$ $i g c_{0}$, and $D=2 \kappa+i(\Delta-\delta)$. Equation (11) becomes

$$
\frac{d}{d t} \vec{V}=-\tilde{M} \cdot \vec{V}+\vec{F}(t),
$$

whose solution can be expressed as [29]

$$
\vec{V}(t)=e^{-\tilde{M}\left(t-t_{0}\right)} \vec{V}\left(t_{0}\right)+\int_{t_{0}}^{t} e^{-\tilde{M}\left(t-t^{\prime}\right)} \vec{F}\left(t^{\prime}\right) d t^{\prime} .
$$

If we take $t_{0} \rightarrow-\infty$, the solution becomes

$$
\vec{V}(t)=\int_{-\infty}^{t} e^{-\tilde{M}\left(t-t^{\prime}\right)} \vec{F}\left(t^{\prime}\right) d t^{\prime}
$$

If $\vec{F}\left(t^{\prime}\right)$ is constant, the steady-state solution $\vec{V}=\widetilde{M}^{-1} \vec{F}$ and $\dot{\vec{V}}=0$.

We take the pump field as constant, whereas the probe field depends on time. After solving Eq. (11) analytically, we find $c_{+}(t)$ and $q_{+}(t)$ in terms of hypergeometric functions, and the final result is plotted in Fig. 9 under the EIT condition of $\Delta=\omega_{m}$. We plot mirror vibrations $q_{+}(t)$ as a function of time in Fig. 9 for $\varepsilon_{p}(t)=\operatorname{sech}(t)$. The total displacement of the robust excitations of NMM is

$$
q(t)=q_{0}+2 q_{+}(t) \cos \delta t .
$$

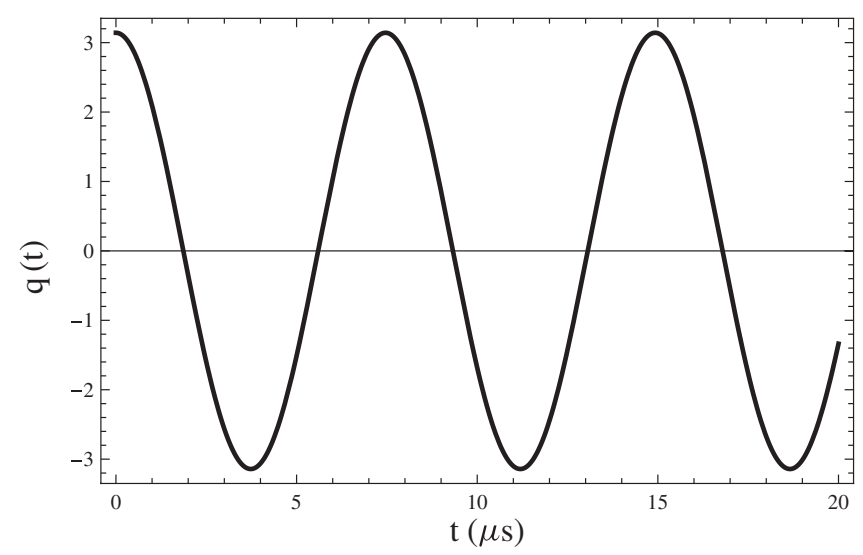

FIG. 10. Time dependence of $q(t)$ for $\Delta=\omega_{m}$ and $\delta=\omega_{m}$. All parameters are the same as those in Fig. 2. 
We find situations with both robust excitations of NMM motional modes, where the mirror is simply displaced thanks to optical intensity. This behavior is displayed in Fig. 10. During the final stages of this work, similar time-dependent control procedures were considered for optomechanical quantum memory applications [15].

\section{CONCLUSION}

We have examined the question of the delay and advance of the probe field under the conditions of electromagnetically induced transparency in an optomechanical system contained in a high-quality double-ended cavity. We have shown that it is possible to control the propagation of the probe pulse in a double-ended cavity with a NNM. We have computed the transmission and reflection spectrums of the probe field. The tunable group delay and the advance of the optical pulse by adjusting the pump power were found. As the pump power increases, the group delay becomes smaller, while it saturates beyond a critical value of the pump power. The magnitude of the group delay is found to be $\sim 1 \mathrm{~ms}$, and the advance is about $-2 \mathrm{~s}$ at a low pump power of $\sim 0.2 \mu \mathrm{W}$ for the parameters chosen as in Ref. [19]. The system under consideration is easier to implement and offers longer group delays compared to other optomechanical proposals [23]. Moreover, we have investigated the interaction of the optomechanical cavity with a time-dependent probe field for controlled excitations of mirror vibrations, and therefore we have showed that thanks to optical intensity, mechanical-mode excitations can be caused by a time-dependent probe field.

\section{ACKNOWLEDGMENTS}

D.T. acknowledges support from the Faculty Support Program of the Council of Higher Education (YÖK) of Turkey. D.T. thanks G. S. Agarwal for many stimulating and fruitful discussions and his hospitality at Oklahoma State University, Stillwater, Oklahoma, USA.
[1] L. V. Hau, S. E. Harris, Z. Dutton, and C. H. Behroozi, Nature (London) 397, 594 (1999).

[2] S. E. Harris, Phys. Today 50(7), 36 (1997).

[3] M. D. Lukin, Rev. Mod. Phys. 75, 457 (2003).

[4] M. Fleischhauer, A. Imamoglu, and J. P. Marangos, Rev. Mod. Phys. 77, 633 (2005).

[5] M. D. Lukin and A. Imamoglu, Phys. Rev. Lett. 84, 1419 (2000).

[6] M. S. Bigelow, N. N. Lepeshkin, and R. W. Boyd, Phys. Rev. Lett. 90, 113903 (2003).

[7] M. Fleischhauer and M. D. Lukin, Phys. Rev. Lett. 84, 5094 (2000).

[8] C. Liu, Z. Dutton, C. H. Behroozi, and L. V. Hau, Nature (London) 409, 490 (2001).

[9] L. J. Wang, A. Kuzmich, and A. Dogariu, Nature (London) 406, 277 (2000).

[10] A. Dogariu and A. Kuzmich, and L. J. Wang, Phys. Rev. A 63, 053806 (2001).

[11] M. S. Bigelow, N. N. Lepeshkin, and R. W. Boyd, Science 301, 200 (2003).

[12] A. H. Safavi-Naeini, T. P. Alegre, J. Chan, M. Eichenfield, M. Winger, Q. Lin, J. T. Hill, D. E. Chang, and O. Painter, Nature (London) 472, 69 (2011).

[13] D. E. Chang, A. H. Safavi-Naeini, M. Hafezi, and O. Painter, New J. Phys 13, 023003 (2011).

[14] G. S. Agarwal and S. Huang, Phys. Rev. A 85, 021801(R) (2012).
[15] K. Qu and G. S. Agarwal, arXiv:1210.4067.

[16] I. Favero and K. Karrai, Nat. Photonics 3, 201 (2009).

[17] A. Schliesser, R. Rivière, G. Anetsberger, O. Arcizet, and T. J. Kippenberg, Nat. Phys. 4, 415 (2008).

[18] S. Gröblacher, K. Hammerer, M. Vanner, and M. Aspelmeyer, Nature (London) 460, 724 (2009).

[19] J. D. Thompson, B. M. Zwickl, A. M. Jayich, F. Marquardt, S. M. Girvin, and J. G. E. Harris, Nature (London) 452, 72 (2008).

[20] M. Bhattacharya and P. Meystre, Phys. Rev. Lett. 99, 073601 (2007).

[21] S. Weis, R. Riviere, S. Deleglise, E. Gavartin, O. Arcizet, A. Schliesser, and T. J. Kippenberg, Science 330, 1520 (2010).

[22] A. Nunnenkamp, K. Borkje, and S. M. Girvin, Phys. Rev. Lett. 107, 063602 (2011).

[23] B. Chen, C. Jiang, and K.-D. Zhu, Phys. Rev. A 83, 055803 (2011).

[24] G. S. Agarwal and S. Huang, Phys. Rev. A 81, 041803(R) (2010).

[25] M. Karuza, C. Biancofiore, C. Molinelli, M. Galassi, R. Natali, P. Tombesi, G. Di Giuseppe, and D. Vitali, arXiv:1209.1352.

[26] D. Tarhan, arXiv:1210.6213.

[27] M. Bhattacharya, H. Uys, and P. Meystre, Phys. Rev. A 77, 033819 (2008).

[28] R. Boyd, Nonlinear Optics (Academic, Amsterdam, 2008).

[29] M. O. Scully and M. S. Zubairy, Quantum Optics (Cambridge University Press, Cambridge, 1997). 